\title{
Playing Muller Games in a Hurry *
}

\author{
John Fearnley \\ Department of Computer Science \\ University of Warwick, UK \\ john@dcs.warwick.ac.uk
}

\author{
Martin Zimmermann \\ Lehrstuhl Informatik 7 \\ RWTH Aachen University, Germany \\ zimmermann@automata.rwth-aachen.de
}

This work studies the following question: can plays in a Muller game be stopped after a finite number of moves and a winner be declared. A criterion to do this is sound if Player 0 wins an infinite-duration Muller game if and only if she wins the finite-duration version. A sound criterion is presented that stops a play after at most $3^{n}$ moves, where $n$ is the size of the arena. This improves the bound $(n !+1)^{n}$ obtained by McNaughton and the bound $n !+1$ derived from a reduction to parity games.

\section{Introduction}

In an infinite game, two players move a token through a finite graph thereby building an infinite path. The winner is determined by a partition of the infinite paths through the arena into the paths that are winning for Player 0 or winning for Player 1, respectively. Many winning conditions in the literature depend on the vertices that are visited infinitely often, i.e., the winner of a play cannot be determined after any finite number of steps. We are interested in the following question: is it nevertheless possible to give a criterion to define a finite-duration variant of an infinite game. Such a criterion has to stop a play after a finite number of steps and then declare a winner based on the finite play constructed thus far. It is sound if Player 0 has a winning strategy for the infinite-duration game if and only if she has a winning strategy for the finite-duration game.

McNaughton considered the problem of playing infinite games in finite time from a different perspective. His motivation was to make infinite games suitable for "casual living room recreation" [8]. As human players cannot play infinitely long, he envisions a referee that stops a play at a certain time and declares a winner. The justification for declaring a winner is that "if the play were to continue with each [player] playing forever as he has so far, then the player declared to be the winner would be the winner of the infinite play of the game" [8].

Besides this recreational aspect of infinite games there are several interesting theoretical questions that motivate investigating this problem. If there exists a sound criterion to stop a play after at most $n$ steps, this yields a simple algorithm to determine the winner of the infinite game: the finite-duration game can be seen as a reachability game on a finite tree of depth at most $n$ that is won by the same player that wins the infinite-duration game. There exist simple and efficient algorithms to determine the winner in reachability games on trees. Furthermore, a positive answer to the question whether a winning strategy for the reachability game can be turned into a (small finite-state) winning strategy should yield better results in the average (although not in the worst case) than game reductions, which ignore the structure of the arena.

Consider the following criterion: the players move the token through the arena until a vertex is visited for the second time. An infinite play can then be obtained by assuming that the players continue to play

\footnotetext{
${ }^{*}$ This work was carried out while the second author visited the University of Warwick, supported by EPSRC grant EP/E022030/1 and the project Games for Analysis and Synthesis of Interactive Computational Systems (GASICS) of the European Science Foundation.
}

GandALF 2010

EPTCS ??, 20??, pp. 1-16 doi 10.4204/EPTCS.??. 
the cycle that they have constructed. Then, the winner of the infinite play is declared to be the winner of the finite play. If the game is determined with positional strategies for both players, then this procedure is correct: if a player has a winning strategy for the infinite game, which can be assumed to be positional, then she can use the same strategy to win the finite version of the game and vice versa.

Therefore, McNaughton proposes that we should consider games that are in general not positionally determined. Here, the first loop of a play is typically not an indicator of how the infinite play evolves, as the memory allows a player to make different decisions when a vertex is seen again. Therefore, the players have to play longer before the play can be stopped and analyzed.

McNaughton considers Muller games, which are games of the form $\left(G, \mathscr{F}_{0}, \mathscr{F}_{1}\right)$, where $G$ is a finite arena and $\left(\mathscr{F}_{0}, \mathscr{F}_{1}\right)$ is a partition of the set of vertices. Player $i$ wins a play, if the set of vertices visited infinitely often by this play is in $\mathscr{F}_{i}$. Muller winning conditions allow us to express all other winning conditions that depend only in the infinity set of a play (e.g., Büchi, co-Büchi, parity, Rabin, and Streett conditions).

To give a sound criterion for Muller games, McNaughton defines for every set of vertices $F$ a scoring function $\mathrm{Sc}_{F}$ that keeps track of the number of times the set $F$ was visited entirely since the last visit of a vertex that is not in $F$. In an infinite play, the set of vertices seen infinitely often is the unique set $F$ such that $\mathrm{Sc}_{F}$ will tend to infinity with being reset to 0 only finitely often.

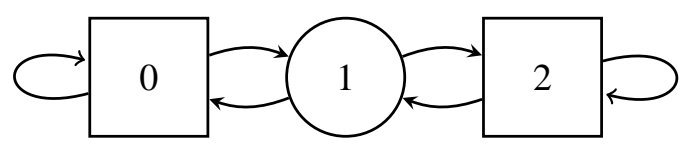

Figure 1: The arena $G$.

Let $G$ be the arena in Figure 1(Player 0's vertices are shown as circles and Player 1's vertices are shown as squares) and the Muller game $\mathscr{G}=\left(G, \mathscr{F}_{0}, \mathscr{F}_{1}\right)$ with $\mathscr{F}_{0}=\{\{0,1,2\},\{0\},\{2\}\}$. In the play 100122121 we have that the score for the set $\{1,2\}$ is 3 , as it was seen thrice (i.e., with the infixes 12 , 21 , and 21). Note that the order of the visits to the elements of $F$ is irrelevant and that it is not required to close a loop in the arena. The following winning strategy for Player 0 bounds the scores of Player 1 by 2: arriving from 0 at 1 move to 2 and vice versa. However, Player 0 cannot avoid a score of 2 for her opponent, as either the play prefix 1001 or 1221 is consistent with every winning strategy.

By using finite-state determinacy of Muller games, McNaughton suggests that the criterion should stop a play after a score of $|F| !+1$ for some set $F$ is reached. He shows that picking the winner to be the Player $i$ such that $F \in \mathscr{F}_{i}$ is indeed sound.

Applying finite-state determinacy one can also show that one can soundly declare a winner after at most $|G| !+1$ steps, as a repetition of a memory state has occurred after that many steps. Note that for large sets $F$, it could take far more than $|G| !+1$ steps to reach a score of $|F| !+1$, as scores can increase slowly or can even be reset to 0 . However, to decide whether a memory state repetition has occurred, it might be necessary to compute the complete memory structure for the given game, which is of size $|G|$ !. Keeping track of scores is much simpler, as they can be computed on the fly while the play is being played. Also, there are at most $|G|$ sets $F$ with non-zero score.

Our contribution. We show that declaring the winner of a play as soon as the score 3 is reached for the first time is a sound criterion. We complement this by proving that a score of 3 is reached after at most $3^{|G|}$ steps. Hence, we obtain a better bound than $|G| !+1$, which was derived from waiting for repetitions of memory states. 
Our results are obtained by using Zielonka's algorithm [10] (a reinterpretation of an earlier algorithm by McNaughton [7]) for computing winning regions in Muller games. We carefully define a winning strategy that bounds the scores of the opponent by 2 . In the example above, the winning player cannot avoid a score of 2 for the opponent. Hence, in this sense our result is optimal.

Related work. Usually, the quality of a strategy is measured in terms of memory needed to implement it. However, there are other natural quality measures of winning strategies. In [2], the authors study a strengthening of parity (and Streett) objectives, which require that there is some bound between the occurrences of even colors. Another application of this concept appears in work on request response games [6, 11], where waiting times between requests and subsequent responses are used to define the value of a play. There it is shown that time-optimal winning strategies can be computed effectively.

The maximal score achieved by the opponent in a play can be used to measure the quality of winning plays in a Muller game. Player 0 prefers plays in which the scores of her opponent are small. This corresponds to not spending a prolonged amount of time in a set of the opponent, but visiting every vertex that is seen infinitely often without large gaps.

This paper is structured as follows. Section 2 contains basic definitions and fixes our notation. In Section 3, we introduce the scoring functions, prove some properties about scoring and define finite-time Muller games. In Section 4 we present Zielonka's algorithm which is used in Section 5 to prove the main result. Section 6 ends the paper with a conclusion and some pointers to further research.

\section{Definitions}

The power set of a set $S$ is denoted by $2^{S}$ and $\mathbb{N}$ denotes the non-negative integers. The prefix relation on words is denoted by $\sqsubseteq$, its strict version by $\sqsubset$. Given a word $w=x y$, define $x^{-1} w=y$ and $w y^{-1}=x$.

An arena $G=\left(V, V_{0}, V_{1}, E\right)$ consists of a finite, directed graph $(V, E)$ of vertices and a partition $\left(V_{0}, V_{1}\right)$ of $V$ denoting the positions of Player 0 (drawn as circles) and Player 1 (drawn as squares). We require that every vertex has at least one outgoing edge. A set $X \subseteq V$ induces the subarena $G[X]=$ $\left(V \cap X, V_{0} \cap X, V_{1} \cap X, E \cap(X \times X)\right)$, if every vertex in $X$ has at least one successor in $X$. A Muller game $\mathscr{G}=\left(G, \mathscr{F}_{0}, \mathscr{F}_{1}\right)$ consists of an arena $G$ and a partition $\left(\mathscr{F}_{0}, \mathscr{F}_{1}\right)$ of $2^{V}$.

A play in $G$ starting in $v \in V$ is an infinite sequence $\rho=\rho_{0} \rho_{1} \rho_{2} \ldots$ such that $\rho_{0}=v$ and $\left(\rho_{n}, \rho_{n+1}\right) \in E$ for all $n \in \mathbb{N}$. The occurrence set $\operatorname{Occ}(\rho)$ and infinity $\operatorname{set} \operatorname{Inf}(\rho)$ of $\rho$ are given by $\operatorname{Occ}(\rho)=\{v \in V \mid \exists n \in$ $\mathbb{N}$ such that $\left.\rho_{n}=v\right\}$ and $\operatorname{Inf}(\rho)=\left\{v \in V \mid \exists^{\omega} n \in \mathbb{N}\right.$ such that $\left.\rho_{n}=v\right\}$. We will also use the occurrence set of a finite play $w$. A play $\rho$ in a Muller game is winning for Player $i$ if $\operatorname{Inf}(\rho) \in \mathscr{F}$.

A strategy for Player $i$ is a function $\sigma: V^{*} V_{i} \rightarrow V$ satisfying $(s, \sigma(w s)) \in E$ for all $w s \in V^{*} V_{i}$. The play $\rho$ is consistent with $\sigma$ if $\rho_{n+1}=\sigma\left(\rho_{0} \ldots \rho_{n}\right)$ for every $n \in \mathbb{N}$ with $\rho_{n} \in V_{i}$. The set of strategies for Player $i$ is denoted by $\Pi_{i}$. A strategy is called finite-state, if it can be implemented by an automaton with output that reads finite plays and outputs the vertex to move to. We will say that a finite-state strategy is of size $n$, if there exists an automaton with $n$ states that implements it.

A strategy $\sigma$ for Player $i$ is a winning strategy from a vertex $v \in V$, if every play that starts in $v$ and is consistent with $\sigma$ is won by Player $i$. The strategy $\sigma$ is a winning strategy for a set of vertices $W \subseteq V$, if every play that starts in some $v \in W$ and is consistent with $\sigma$ is won by Player $i$. The winning region $W_{i}$ of Player $i$ contains all vertices, from which she has a winning strategy. A game is determined if $W_{0}$ and $W_{1}$ form a partition of $V$.

Theorem 1 ([1 5 7]). Muller games are determined with finite-state strategies of size $n \cdot n !$, where $n$ denotes the size of the arena. 
Let $G=\left(V, V_{0}, V_{1}, E\right)$ be an arena and let $X \subseteq V$ be a set that induces a subarena. The attractor for Player $i$ of a set $F \subseteq V$ in $X$ is $\operatorname{Attr}_{i}^{X}(F)=\bigcup_{n=0}^{|V|} A_{n}$ where $A_{0}=F \cap X$ and

$$
\begin{aligned}
A_{n+1}=A_{n} & \cup\left\{v \in V_{i} \cap X \mid \exists v^{\prime} \in A_{n} \text { such that }\left(v, v^{\prime}\right) \in E\right\} \\
& \cup\left\{v \in V_{1-i} \cap X \mid \forall v^{\prime} \in X \text { with }\left(v, v^{\prime}\right) \in E: v^{\prime} \in A_{n}\right\} .
\end{aligned}
$$

A $X \subseteq V$ is a trap for Player $i$, if all outgoing edges of the vertices in $V_{i} \cap X$ lead to $X$ and at least one successor of every vertex in $V_{1-i} \cap X$ is in $X$.

Lemma 2. Let $G=\left(V, V_{0}, V_{1}, E\right)$ be an arena and $F, X \subseteq V$.

1. For every $v \in \operatorname{Attr}_{i}^{X}(F)$ Player $i$ has a positional strategy to bring the play into $F$.

2. The set $V \backslash \operatorname{Attr}_{i}^{X}(F)$ induces a subarena and is a trap for Player i in $G$.

\section{The Scoring Functions and Finite-time Muller Games}

This section introduces the notions that are required to formally define finite-time Muller games. In his study of these games, McNaughton introduced the concept of a score. For every set of vertices $F$ we define the score of a finite play $w$ to be the number of times that $F$ has been visited entirely since $w$ last visited a vertex in $V \backslash F$.

Definition 3 (Score). For every $F \subseteq V$ we define $\mathrm{Sc}_{F}: V^{+} \rightarrow \mathbb{N}$ as

$$
\operatorname{Sc}_{F}(w)=\max \left\{k \in \mathbb{N} \mid \exists x_{1}, \ldots, x_{k} \in V^{+} \text {such that } \operatorname{Occ}\left(x_{i}\right)=F \text { for all } i \text { and } x_{1} \cdots x_{k} \text { is a suffix of } w\right\} .
$$

We extend this notion by introducing the concept of an accumulator. For every set $F$, the accumulator measures the progress that has been made towards the next score increase of $F$.

Definition 4 (Accumulator). For every $F \subseteq V$ we define $\operatorname{Acc}_{F}: V^{+} \rightarrow 2^{F}$ by $\operatorname{Acc}_{F}(w)=\operatorname{Occ}(x)$, where $x$ is the longest suffix of $w$ such that $\operatorname{Sc}_{F}(w)=\operatorname{Sc}_{F}\left(w y^{-1}\right)$ for every suffix $y$ of $x$, and $\operatorname{Occ}(x) \subseteq F$.

Finally we define the maximum score function. This function maps a subset $\mathscr{F} \subseteq 2^{V}$ and a play $\rho$ to the highest score that is reached during $\rho$ for a set contained in $\mathscr{F}$.

Definition 5 (MaxScore). For every $\mathscr{F} \subseteq 2^{V}$ we define $\operatorname{MaxSc}_{\mathscr{F}}: V^{+} \cup V^{\omega} \rightarrow \mathbb{N} \cup\{\infty\}$ by $\operatorname{MaxSc}_{\mathscr{F}}(\rho)=$ $\max _{F \in \mathscr{F}} \max _{w \sqsubseteq \rho} \operatorname{Sc}_{F}(w)$.

McNaughton proposes that scores should be used to decide the winner in a finite-time Muller game. As soon as a threshold score of $k$ for some set $F$ is reached, the play is stopped and Player $i$ is declared the winner, if $F \in \mathscr{F}_{i}$. The next lemma shows that this condition is sufficient to ensure that the game terminates after a finite number of steps.

Lemma 6. Let $G$ be an arena with vertex set $V$. Every $w \in V^{*}$ with $|w| \geq k^{|V|}$ satisfies $\operatorname{MaxSc}_{2^{V}}(w) \geq k$.

Proof. We will show by induction over $|V|$ that every word $w \in V^{*}$ with $|w| \geq k^{|V|}$ contains an infix $x$ that can be decomposed as $x=x_{1} \cdots x_{k}$ where every $x_{i}$ is a non-empty word with $\operatorname{Occ}\left(x_{i}\right)=\operatorname{Occ}(x)$. This will imply $\operatorname{MaxSc}_{2^{v}}(w) \geq k$.

The claim holds trivially for $|V|=1$ by choosing $x$ to be the prefix of $w$ of length $k$ and $x_{i}=s$ for the single vertex $s \in V$. For the induction step, consider a set $V$ with $n+1$ vertices. If $w$ contains an infix $x$ of length $k^{n}$ which contains at most $n$ distinct vertices, then we can apply the inductive hypothesis and obtain a decomposition of an infix of $v$ with the desired properties. Otherwise, every infix $x$ of $w$ of length $k^{n}$ contains every vertex of $V$ at least once. Let $x$ be the prefix of length $k^{n+1}$ of $w$ and let $x=x_{1} \cdots x_{k}$ be the decomposition of $x$ such that each $x_{i}$ is of length $k^{n}$. Then, we have $\operatorname{Occ}\left(x_{i}\right)=\operatorname{Occ}(x)=V$ for all $i$. Therefore, the decomposition has the desired properties. 
Lemma 6 implies that a finite-time Muller game with threshold $k$ must end after at most $k^{|V|}$ steps. We can also show that this bound is tight. For every $k>0$ we give an inductive definition of a word over the alphabet $\Sigma_{n}=\{1, \ldots, n\}$ by $w_{(k, 1)}=1^{k-1}$ and $w_{(k, n)}=\left(w_{(k, n-1)} n\right)^{k-1} w_{(k, n-1)}$. Clearly, the word $w_{(k, n)}$ has length $k^{n}-1$, and it can also be shown that $\operatorname{Max}_{\operatorname{Sc}_{2} \Sigma_{n}}(w)<k$.

Finally, to declare a unique winner in every finite-time Muller game we must exclude the case where there are two sets such that both sets hit score $k$ at the same time. McNaughton observed that for $k \geq 2$ the first set to hit score $k$ will be unique. Before we reprove this, we will first show a useful auxiliary result that will also be used later in the paper.

Lemma 7 (cf. Theorem 4.2 of [8]). Let $w \in V^{+}$. The sets $F$ with $\operatorname{Sc}_{F}(w) \geq 1$ together with the sets $\operatorname{Acc}_{F}(w)$ for some $F$ form a chain with respect to the subset relation.

Proof. It suffices to show that all such sets are pairwise comparable: let $F$ and $F^{\prime}$ be two sets such that either $\operatorname{Sc}_{F}(w) \geq 1$ or $F=\operatorname{Acc}_{H}(w)$ for some $H \subseteq V$ and either $\operatorname{Sc}_{F^{\prime}}(w) \geq 1$ or $F^{\prime}=\operatorname{Acc}_{H^{\prime}}(w)$ for some $H^{\prime} \subseteq V$. Then, there exist two decompositions $w=w_{0} w_{1}$ and $w=w_{0}^{\prime} w_{1}^{\prime}$ with $\operatorname{Occ}\left(w_{1}\right)=F$ and $\operatorname{Occ}\left(w_{1}^{\prime}\right)=F^{\prime}$. Now, either $w_{1}$ is a suffix of $w_{1}^{\prime}$ or vice versa. In the first case, we have $F \subseteq F^{\prime}$ and in the second case $F^{\prime} \subseteq F$.

Note that Lemma[7implies that there are at any time at most $|V|$ sets with non-zero scores.

Lemma 8 ([[8]). Let $k, l \geq 2$, let $F, F^{\prime} \subseteq V$, let $w \in V^{*}$ and $v \in V$ such that $\operatorname{Sc}_{F}(w)<k$ and $\operatorname{Sc}_{F^{\prime}}(w)<l$. If $\operatorname{Sc}_{F}(w v)=k$ and $\operatorname{Sc}_{F^{\prime}}(w v)=l$, then $F=F^{\prime}$.

Proof. Towards a contradiction assume $F \neq F^{\prime}$. By Lemma 7 we can assume $F^{\prime} \subset F$, i.e., there exists some $q \in F \backslash F^{\prime}$. Then, $\mathrm{Sc}_{F}(w v)=k$ and $\mathrm{Sc}_{F^{\prime}}(w v)=l$ imply the existence of decompositions $w v=$ $w_{0} w_{1} \cdots w_{k}$ and $w v=w_{0}^{\prime} w_{1}^{\prime} \cdots w_{l}^{\prime}$ such that $\operatorname{Occ}\left(w_{i}\right)=F$ and $\operatorname{Occ}\left(w_{i}^{\prime}\right)=F^{\prime}$ for all $i \geq 1$. As $q \notin F^{\prime}$, $w_{1}^{\prime} \cdots w_{l}^{\prime}$ is a proper suffix of $w_{k}$. Furthermore, as $\operatorname{Sc}_{F}(w)<k$, we have $v \notin \operatorname{Occ}\left(w_{k} v^{-1}\right)$. However, we have $v \in F^{\prime}$ and hence $v \in \operatorname{Occ}\left(w_{k-1}^{\prime}\right)$, which is an infix of $w_{k} v^{-1}$. This yields the desired contradiction.

We are now in a position to define a finite-time Muller game. Such a game $\mathscr{G}=\left(G, \mathscr{F}_{0}, \mathscr{F}_{1}, k\right)$ consists of an arena $G=\left(V, V_{0}, V_{1}, E\right)$, a partition $\left(\mathscr{F}_{0}, \mathscr{F}_{1}\right)$ of $2^{V}$, and a threshold $k \geq 2$. By Lemma 6 we have that every infinite play must reach score $k$ for some set $F$ after a bounded number of steps. Therefore, we define a play for the finite-time Muller game to be a finite path $w=w_{0} \cdots w_{n}$ with $\operatorname{MaxSc}_{2^{V}}\left(w_{0} \cdots w_{n}\right)=k$, but $\operatorname{MaxSc}_{2^{V}}\left(w_{0} \cdots w_{n-1}\right)<k$. Due to Lemma 8 there is a unique $F \subseteq V$ such that $\operatorname{Sc}_{F}(w)=k$. Player 0 wins the play $w$ if $F \in \mathscr{F}_{0}$ and Player 1 wins otherwise. The definitions of strategies, plays, and winning sets can be redefined for the finite games.

Zermelo [9] has shown that a game in which every play is finite is determined. Therefore, it immediately follows that finite Muller games are determined.

Lemma 9. Finite-time Muller games are determined.

In fact, McNaughton considered a slightly different definition of a finite-time Muller game. Rather than stopping the play when the score of a set reaches the global threshold $k$, his version stops the play when the score of a set $F$ reaches $|F| !+1$.

Theorem $10([8])$. If $W_{i}$ is the winning region of Player $i$ in a Muller game $\left(G, \mathscr{F}_{0}, \mathscr{F}_{1}\right)$, and $W_{i}^{\prime}$ is the winning region of Player i in McNaughton's finite-time Muller game, then $W_{i}=W_{i}^{\prime}$. 


\section{Zielonka's Algorithm For Muller Games}

This section presents Zielonka's algorithm for Muller games [10], a reinterpretation of an earlier algorithm due to McNaughton [7]. Our notation mostly follows [3, 4]. We will use the internal structure of the winning regions as computed by the algorithm to define a strategy that bounds the scores of the losing player by 2 .

As we consider uncolored arenas, we have to deal with Muller games where $\left(\mathscr{F}_{0}, \mathscr{F}_{1}\right)$ is a partition of $2^{V^{\prime}}$ for some finite set $V^{\prime} \supseteq V$, as the algorithm makes recursive calls for such games. This does not change the semantics of Muller games, as we have $\operatorname{Inf}(\rho) \subseteq V$ for every infinite play $\rho$.

We begin by introducing Zielonka trees, a representation of winning conditions $\left(\mathscr{F}_{0}, \mathscr{F}_{1}\right)$. Given a family of sets $\mathscr{F} \subseteq 2^{V^{\prime}}$ and $X \subseteq V^{\prime}$, we define $\mathscr{F} \mid X=\{F \in \mathscr{F} \mid F \subseteq X\}$. Given a partition $\left(\mathscr{F}_{0}, \mathscr{F}_{1}\right)$ of $2^{V^{\prime}}$, we define $\left.\left(\mathscr{F}_{0}, \mathscr{F}_{1}\right)\right\rceil X=\left(\mathscr{F}_{0}\left\lceil X, \mathscr{F}_{1} \uparrow X\right)\right.$. Note that $\left.\mathscr{F}\right\rceil X \subseteq \mathscr{F}$.

Definition 11 (Zielonka tree). For every winning condition $\left(\mathscr{F}_{0}, \mathscr{F}_{1}\right)$ defined over a set $V^{\prime}$, its Zielonka tree $\mathscr{Z}_{\mathscr{F}_{0}, \mathscr{F}_{1}}$ is defined as follows: suppose that $V^{\prime} \in \mathscr{F}_{i}$ and let $V_{0}^{\prime}, V_{1}^{\prime}, \ldots, V_{k-1}^{\prime}$ be the $\subseteq$-maximal sets in $\mathscr{F}_{1-i}$. The tree $\mathscr{Z}_{\mathscr{F}_{0}, \mathscr{F}_{1}}$ consists of a root vertex labelled by $V^{\prime}$ with $k$ children which are defined by

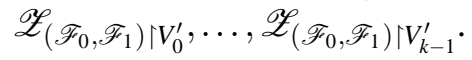

For every Zielonka tree $T$, we define $\operatorname{RtLbl}(T)$ to be the label of the root in $T$, we define $\operatorname{BrnchFctr}(T)$ to be the number of children that the root has in $T$, and we define $\operatorname{Chld}(T, j)$ for $0 \leq j<\operatorname{BrnchFctr}(T)$ to be the $j$-th child of the root in $T$. Here, we assume that the children of every vertex are ordered by some fixed linear order.

The input of Zielonka's algorithm (see Algorithm 10 is a finite arena $G$ with vertex set $V$ and the Zielonka tree of a partition $\left(\mathscr{F}_{0}, \mathscr{F}_{1}\right)$ of $2^{V^{\prime}}$ for some finite set $V^{\prime} \supseteq V$. The algorithm computes the winning regions of the players by successively removing parts of Player 0's winning region (the sets $\left.U_{0}, U_{1}, U_{2}, \ldots\right)$. By doing this, the algorithm computes an internal structure of the winning regions that will be crucial to proving our results in the next section.

For the rest of this paper we will refer to the sets of vertices and the subtrees of $\mathscr{Z}_{\mathscr{F}_{0}, \mathscr{F}_{1}}$ as computed by the algorithm.

Figure 2 depicts the situation in the $n$-th iteration of the algorithm. The vertices in $U_{n-1}$ have already been removed and belong to $W_{1-i}$. Then, all vertices in the $(1-i)$-attractor of $U_{n-1}$ also belong to $W_{1-i}$. After removing these vertices from the arena, the algorithm also removes the vertices in the $i$-attractor of $\operatorname{RtLbl}\left(T_{n}\right)$. The remaining vertices form a subarena whose vertex set is a subset of $\operatorname{RtLbl}\left(T_{n}\right)$. Hence, the algorithm can recursively compute the winning regions $W_{i}^{n}$ in this subarena with Zielonka tree $T_{n}$. By construction, the winning region $W_{1-i}^{n}$ is also a subset of $W_{1-i}$. This is repeated until the sets $U_{n}$ converge to $W_{1-i}$. All remaining vertices belong to $W_{i}$.

Furthermore, we have the following properties that will be used in the next section. Let $n$ denote the index at which Zielonka's algorithm terminated. The sets $W_{1-i}^{j}$ for $j \leq n$ are obviously disjoint. However, the sets $W_{i}^{n-j}$ for $j$ in the range $n-k<j \leq n$ might overlap. Player $i$ can confine a play in $W_{i}^{n-j}$ until Player $1-i$ decides to leave this set. However, his only choice is to move to a vertex in $\operatorname{Attr}_{i}{ }^{X_{n-j}}\left(V \backslash \operatorname{RtLbl}\left(T_{n-j}\right)\right)$, as he can neither move to a vertex in $A_{n}=A_{n-j}\left(X_{n}=X_{n-j}\right.$ is a trap for him) nor to a vertex in $W_{1-i}^{n-j}=\emptyset$. This implies that Player $i$ can force the play to visit $V \backslash \operatorname{RtLbl}\left(T_{n-j}\right)$, if Player $1-i$ decides to leave $W_{1}^{n-j}$.

Theorem 12 ([10]). Algorithm $\square$ terminates with a partition $\left(W_{0}, W_{1}\right)$, where Player 0 has a winning strategy for $W_{0}$ and Player 1 has a winning strategy for $W_{1}$.

Zielonka's winning strategies are defined inductively: Player $1-i$ plays an attractor strategy to $U_{n-1}$ on $A_{n} \backslash U_{n-1}$ and on each $W_{1-i}^{n}$ according to the winning strategy computed recursively. A play consistent 


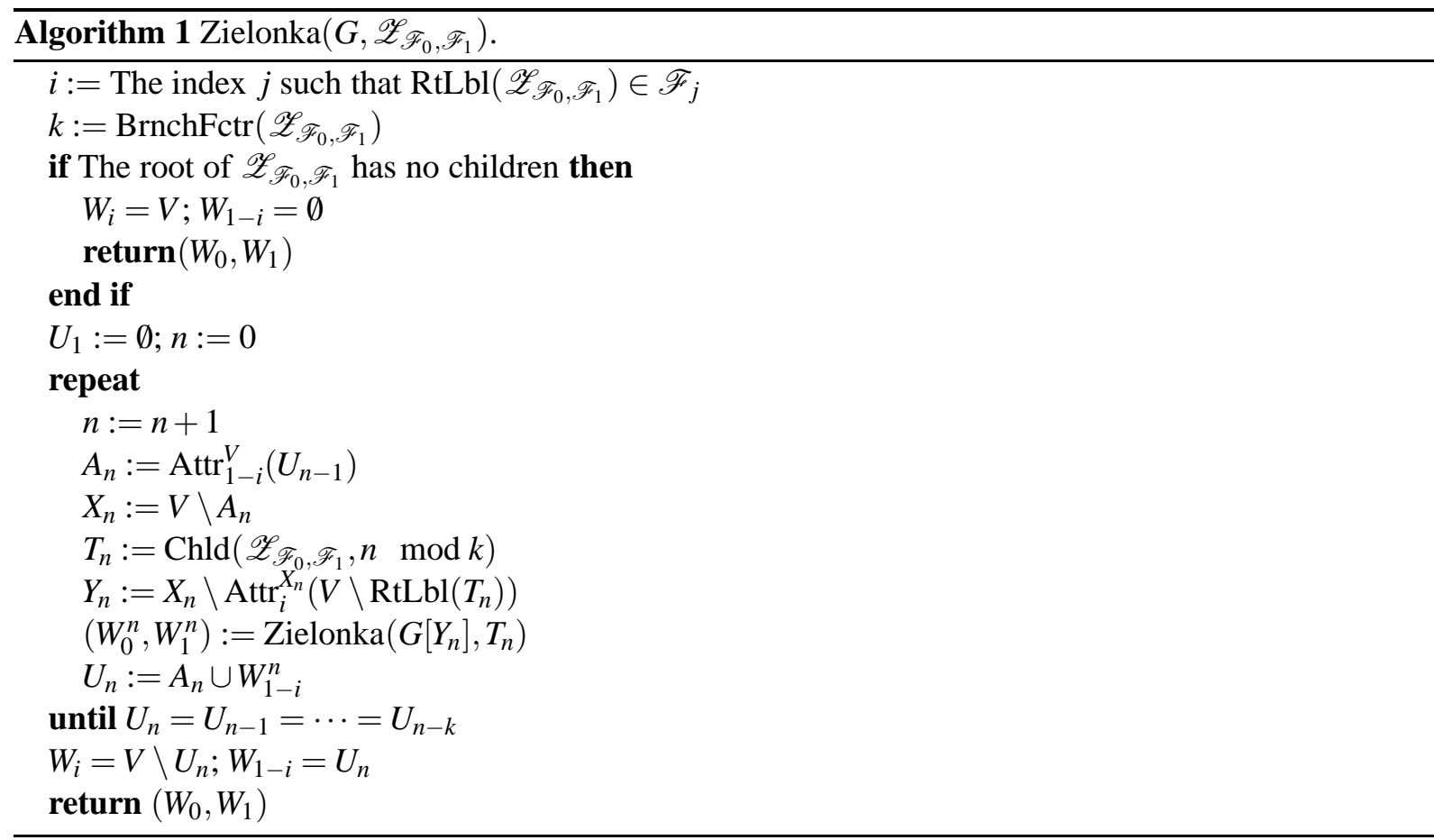

with this strategy will from some point onwards be consistent with one of the winning strategies for some $W_{1-i}^{n}$, hence it is winning for Player $1-i$.

Player $i$ plays using a cyclic counter $c$ : suppose $c=j$. In $W_{1}^{n-j}$, she plays according to the winning strategy computed recursively. If Player $1-i$ chooses to leave $W_{1}^{n-j}$, then she starts playing an attractor strategy to reach $V \backslash \operatorname{RtLbl}\left(T_{n-j}\right)$. Once she has reached this set she increments $c$ modulo $k$ and begins again. There are two possibilities for a play consistent with this strategy: if it stays from some point onwards in some $W_{i}^{n-j}$, then it is winning by the inductive hypothesis. Otherwise, it will visit infinitely many vertices in $V \backslash \operatorname{RtLbl}\left(\operatorname{Chld}\left(\mathscr{Z}_{\mathscr{F}_{0}, \mathscr{F}_{1}}, j\right)\right)$ for every $j$ in the range $0 \leq j<\operatorname{BrnchFctr}\left(\mathscr{Z}_{\mathscr{F}_{0}, \mathscr{F}_{1}}\right)$, which implies that the infinity set of the play is not a subset of any $\operatorname{RtLbl}\left(\operatorname{Chld}\left(\mathscr{Z}_{\mathscr{F}_{0}, \mathscr{F}_{1}}, j\right)\right)$. Hence, it is in $\mathscr{F}_{i}$ and the play is indeed winning for Player $i$.

We conclude this section by showing that the winning strategies for Muller games as defined in [10] do not bound the score of the opponent by a constant.

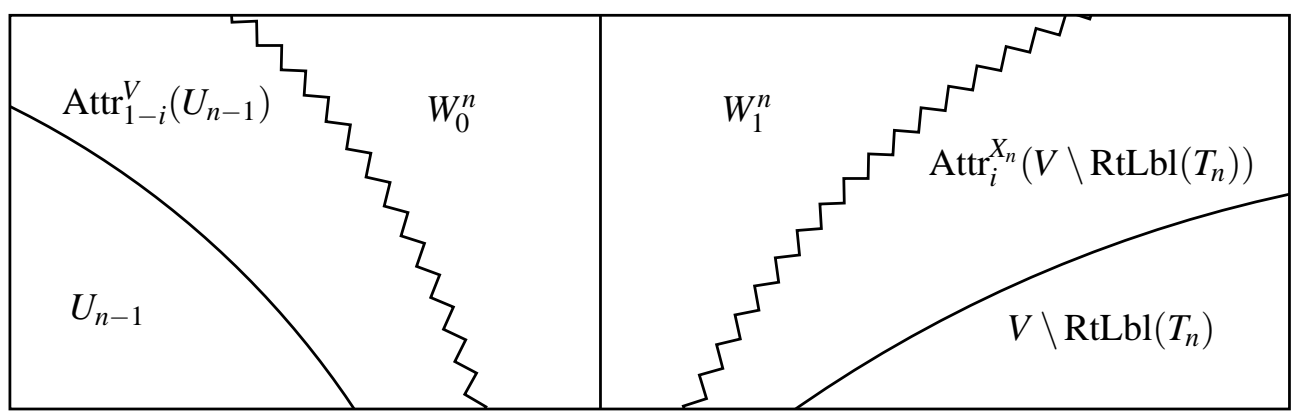

Figure 2: The sets computed by Zielonka's algorithm. 
Lemma 13. There exists a family of Muller games $\mathscr{G}_{n}=\left(G_{n}, \mathscr{F}_{0}^{n}, \mathscr{F}_{1}^{n}\right)$ with $\left|G_{n}\right|=n+1$ and $\left|\mathscr{F}_{0}^{n}\right|=1$ such that $\operatorname{MaxSc}_{\mathscr{F}_{1}^{n}}(\operatorname{Play}(v, \sigma, \tau))=n$ where $\sigma$ is Zielonka's strategy, $v \in V$, and $\tau \in \Pi_{1}$.

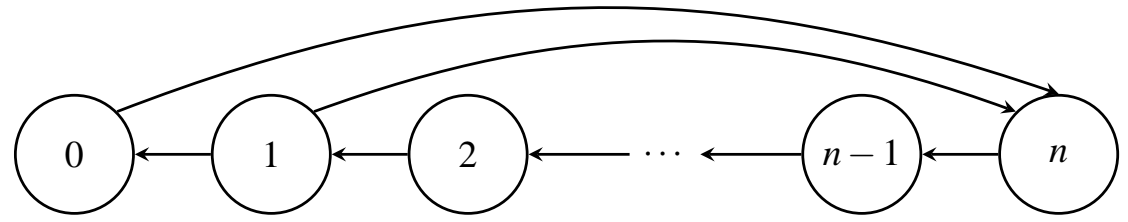

Figure 3: The arena $G_{n}$ for Lemma 13.

Proof. Let $G_{n}=\left(V_{n}, V_{n}, \emptyset, E_{n}\right)$ with $V_{n}=\{0, \ldots, n\}, E_{n}=\{(i+1, i) \mid i<n\} \cup\{(0, n),(1, n)\}$ (see Figure 3), and $\mathscr{F}_{0}^{n}=\left\{V_{n}\right\}$. The Zielonka tree for the winning condition $\left(\mathscr{F}_{0}^{n}, \mathscr{F}_{1}^{n}\right)$ has a root labeled by $V_{n}$ and $n+1$ children that are leaves and are labeled by $V_{n} \backslash\{i\}$ for every $i \in V_{n}$. Assume, the children are ordered as follows: $V_{n} \backslash\{0\}<\cdots<V_{n} \backslash\{n\}$. Zielonka's strategy for $\mathscr{G}_{n}$, which depends on the ordering of the children, can be described as follows. Initialize a counter $c:=0$ and repeat the following:

1. Use an attractor strategy to move to vertex $c$.

2. Increment $c$ modulo $n+1$.

3. Go to 1

Now assume a play consistent with this strategy has just visited 0 . Then, it visits all vertices $1, \ldots, n$ in this order by cycling through the loop $n, \ldots, 1 n$ times. Hence, the score for the set $\{1, \ldots, n\}$ is infinitely often $n$.

By contrast, Player 0 has a positional winning strategy for $\mathscr{G}_{n}$ that bounds the opponents scores by 2 . The reason the strategy described above allows a high score for Player 1 is that it ignores the fact that, while it attracts the play to the vertex 0 , it visits all other vertices. In the next section we will construct a strategy that recognizes such visits. Thereby, the strategy is able to bound the opponent's scores by 2 .

\section{Bounding the Scores in a Muller Game}

In this section, we prove our main result: the finite-time Muller game with threshold 3 is equivalent to a Muller game.

Theorem 14. If $W_{i}$ is the winning region of Player $i$ in a Muller game $\left(G, \mathscr{F}_{0}, \mathscr{F}_{1}\right)$, and $W_{i}^{\prime}$ is the winning region of Player $i$ in the finite-time Muller game $\left(G, \mathscr{F}_{0}, \mathscr{F}_{1}, 3\right)$, then $W_{i}=W_{i}^{\prime}$.

To prove Theorem 14 we use the following approach. If $\operatorname{MaxSc}_{\mathscr{F}_{1-i}}(\rho) \leq 2$ for an infinite play $\rho$, then there exists a prefix $w$ of $\rho$ that is winning for Player $i$ in the finite-time Muller game with threshold 3. Hence, if a winning strategy for Player $i$ in the Muller game bounds the scores of her opponent by 2, then this strategy is also winning for the finite-time Muller game with threshold 3. We will show that such a winning strategy exists. Theorem 14 then follows by determinacy of Muller games. Therefore, the rest of this section will be dedicated to proving the following lemma.

Lemma 15. Player $i$ has a winning strategy $\sigma$ for her winning region $W_{i}$ in a Muller game $\mathscr{G}=$ $\left(G, \mathscr{F}_{0}, \mathscr{F}_{1}\right)$ such that $\operatorname{MaxSc}_{\mathscr{F}_{1-i}}(\operatorname{Play}(v, \sigma, \tau)) \leq 2$ for every vertex $v \in W_{i}$ and every $\tau \in \Pi_{1-i}$. 
We will use the internal structure of the winning regions as computed by Zielonka's algorithm to give an inductive proof of this claim. Traditionally, Zielonka's strategies forget the history of the play every time they switch between an attractor strategy and a recursively computed winning strategy. For example, suppose that a play $w$ spends some time in $W_{1}^{n-j}$ before Player 0 decides to move out of the set $W_{1}^{n-j}$. Player 1 responds to this by playing the attractor strategy to the set $V \backslash \operatorname{RtLbl}\left(T_{n-j}\right)$ in order to reach some vertex $v \in V \backslash \operatorname{RtLbl}\left(T_{n-j}\right)$. If $v \in W_{1}^{n-j+1}$, then Player 1 will play the winning strategy for the set $W_{1}^{n-j+1}$ starting at the vertex $v$.

Note that the play $w$ may have spent a significant number of steps in $W_{1}^{n-j+1}$ (while playing according to the attractor strategy) before Player 1 begins to play the winning strategy for that set. Yet in Zielonka's strategy, Player 1 will behave as if the first vertex visited in $W_{1}^{n-j+1}$ is $v$. In other words, the suffix of $w$ that is contained in $W_{1}^{n-j+1}$ is effectively forgotten by the strategy.

This fact is irrelevant if we are only concerned with constructing a winning strategy, but when we want to construct strategies that guarantee certain scores are bounded by 2 , the entire suffix of $w$ must be retained in this kind of situation. This motivates the following definition of a play. A play begins with a finite prefix over which the players have no control, and then continues as a normal play would. The key difference is that the strategies may base their decisions on the properties of the prefix.

Definition 16 (Play). For a non-empty finite path $w=w_{0} \cdots w_{n}$ and strategies $\sigma \in \Pi_{i}, \tau \in \Pi_{1-i}$, we define the infinite play Play $(w, \sigma, \tau)=\rho_{0} \rho_{1} \rho_{2} \cdots$ inductively by $\rho_{j}=w_{j}$ for $0 \leq j \leq n$ and for $j>n$ by

$$
\rho_{j}=\left\{\begin{array}{ll}
\sigma\left(\rho_{0} \cdots \rho_{j-1}\right) & \text { if } \rho_{j-1} \in V_{i} \\
\tau\left(\rho_{0} \cdots \rho_{j-1}\right) & \text { if } \rho_{j-1} \in V_{1-i}
\end{array} .\right.
$$

In fact, the finite paths that are passed to our strategies will not be totally arbitrary. As described previously, these paths arise out of decisions made before the strategy was recursively applied. Therefore, we have some control over the form that these paths take. We will construct our strategy so that every path passed to a recursive strategy has the following property.

Definition 17 (Burden). Let $\mathscr{F} \subseteq 2^{V^{\prime}}$. A finite path $w$ is an $\mathscr{F}$-burden if $\operatorname{MaxSc}_{\mathscr{F}}(w) \leq 2$ and for every $F \in \mathscr{F}$ either $\operatorname{Sc}_{F}(w)=0$ or $\operatorname{Sc}_{F}(w)=1$ and $\operatorname{Acc}_{F}(w)=\emptyset$.

We are now ready to prove by induction over the height of the Zielonka tree that both players have a strategy to bound their opponent's scores by 2 on their winning regions, even if the play starts with a burden. We begin by considering the base case, which is when the Zielonka tree is a leaf. For the rest of this section we will assume $\operatorname{RtLbl}\left(\mathscr{Z}_{\mathscr{F}_{0}, \mathscr{F}_{1}}\right) \in \mathscr{F}_{1}$. Otherwise, swap the roles of Player 0 and 1 below.

Lemma 18. Let $\left(G, \mathscr{F}_{0}, \mathscr{F}_{1}\right)$ be a Muller game with vertex set $V$ such that $\mathscr{Z}_{\mathscr{F}_{0}, \mathscr{F}_{1}}$ is a leaf. Then, Player 1 has a strategy $\tau$ such that $\operatorname{MaxSc}_{\mathscr{F}_{0}}(\operatorname{Play}(w v, \sigma, \tau)) \leq 2$ for every strategy $\sigma \in \Pi_{0}$ and every $\mathscr{F}_{0}$-burden wv with $v \in V$.

Proof. As $\mathscr{Z}_{\mathscr{F}_{0}, \mathscr{F}_{1}}$ is a leaf and $\operatorname{Rt} \operatorname{Lbl}\left(\mathscr{Z}_{\mathscr{F}_{0}, \mathscr{F}_{1}}\right) \in \mathscr{F}_{1}$ by assumption, we have $\mathscr{F}_{0}=\emptyset$. Hence, any strategy $\tau$ for Player 1 guarantees $\operatorname{MaxSc}_{\mathscr{F}_{0}}(\operatorname{Play}(w, \sigma, \tau)) \leq 2$.

We now move on to the inductive step of the proof. We will give two versions of the inductive step, one case will be for the set $W_{0}$ and the other will be for the set $W_{1}$. We will consider the case for the set $W_{0}$ first.

The situation in this case is shown in Figure 4 Our strategy for this case will be the same as Zielonka's strategy, but it must also deal with the finite path that has been passed to it. We denote the attractor strategy for Player 0 on $A_{n} \backslash U_{n-1}$ by $\sigma_{n}^{\mathrm{A}}$ and we denote the recursively computed strategy 


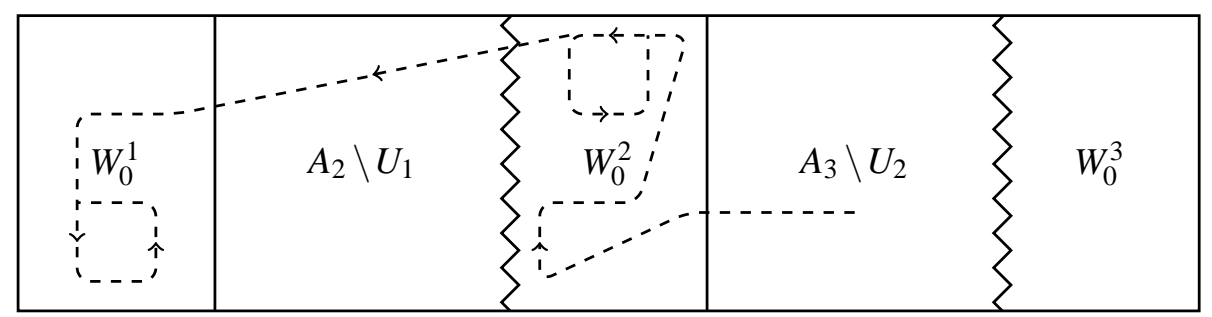

Figure 4: The structure of $W_{0}$. The dashed line indicates a play.

for Player 0 on $G\left[W_{0}^{n}\right]$ as $\sigma_{n}^{\mathrm{R}}$. We can assume that $\sigma_{n}^{\mathrm{R}}$ satisfies the inductive hypothesis, which means that $\operatorname{MaxSc}_{\mathscr{F}_{1}\left\lceil W_{0}^{n}\right.}\left(\operatorname{Play}\left(w v, \sigma_{n}^{\mathrm{R}}, \tau\right)\right) \leq 2$ for every strategy $\tau$ for Player 1 in $G\left[W_{0}^{n}\right]$ and every $\mathscr{F}_{1}\left\lceil W_{0}^{n}\right.$-burden $w v$ with $v \in W_{0}^{n}$. We define the following strategy $\sigma^{*}$ for $W_{0}$ :

$$
\sigma^{*}(w v)=\left\{\begin{array}{ll}
\sigma_{n}^{\mathrm{R}}\left(w^{\prime} v\right) & \text { if } v \in W_{0}^{n} \text { and } w^{\prime} \text { is the longest suffix of } w \text { with } \operatorname{Occ}\left(w^{\prime}\right) \subseteq W_{0}^{n} \\
\sigma_{n}^{\mathrm{A}}(v) & \text { if } v \in A_{n} \backslash U_{n-1}
\end{array} .\right.
$$

Our strategy chooses to use $\sigma_{n}^{\mathrm{R}}$ or $\sigma_{n}^{\mathrm{A}}$ precisely when Zielonka's strategy chooses to do so. The difference is that our strategy is careful to pass the appropriate finite path to the recursively computed strategy $\sigma_{n}^{\mathrm{R}}$.

The sets $U_{j}$ form a sequence of nested traps for Player 1. Therefore, if Player 1 chooses to leave some $U_{j} \backslash U_{j-1}$ and Player 0 plays according to $\sigma^{*}$, the play can never return to $U_{j} \backslash U_{j-1}$. This implies that a play that has left some $W_{0}^{j}$ will never return. Also, every vertex in $A_{j} \backslash U_{j-1}$ can be seen at most once, as $\sigma^{*}$ behaves like an attractor strategy on these vertices. The next lemma will be used to deal with cases that arise from these observations.

Lemma 19. Let $w$ be an $\{F\}$-burden, let $v, v^{\prime} \in F$.

1. Let $\rho$ be an infinite play in which $v$ appears at most once. Then, $\operatorname{MaxSc}_{\{F\}}(w \rho) \leq 2$.

2. Let $\rho$ be an infinite play such that $v$ is never visited after $v^{\prime}$ was visited for the first time. Then, $\operatorname{MaxSc}_{\{F\}}(w \rho) \leq 2$.

Proof. For both statements, it suffices to show that $\mathrm{Sc}_{F}(w x) \leq 2$ for every prefix $x$ of $\rho$. Let $w=w_{0} \cdots w_{n}$. We consider the two cases given by the definition of a burden:

- $\operatorname{Sc}_{F}(w)=1$. As $\operatorname{Acc}_{F}(w)=\emptyset$, we have $\operatorname{Sc}_{F}\left(w_{0} \cdots w_{n-1}\right)=0$. Hence, the suffix $w_{k} \cdots w_{n}$ of $w$ witnessing $\operatorname{Sc}_{F}(w)=1$ is minimal.

1. As $w_{k} \cdots w_{n}$ is minimal and as $v$ occurs at most once in $\rho$, we conclude that the score for $F$ increases at most once after the prefix $w$.

2. As the suffix is minimal, the score of $F$ can increase to 2 only by or after visiting $v^{\prime}$ for the first time. But $v$ is then never visited again. Hence, the score for $F$ is bounded by 2 .

- $\operatorname{Sc}_{F}(w)=0$. Let $y$ be the shortest prefix of $\rho$ such that $\operatorname{Sc}_{F}(w y)=1$. If such a prefix does not exist, then we are done.

1. Otherwise, $y^{-1} \rho$ does contain $v$ at most once. Hence, the score for $F$ increases at most once after the prefix $w y$.

2. Again, if such a prefix exists, then the score for $F$ can reach 2 only by or after visiting $v^{\prime}$ for the first time after $w y$. But $v$ is then never visited again. Hence, the score for $F$ is bounded by 2 . 
We are now able to prove the inductive step for Player 0, by applying the the observations formalized in Lemma 19 to the structure of $W_{0}$.

Lemma 20. We have $\operatorname{MaxSc}_{\mathscr{F}_{1}\left\lceil W_{0}\right.}\left(\operatorname{Play}\left(w v, \sigma^{*}, \tau\right)\right) \leq 2$ for every strategy $\tau \in \Pi_{1}$ and every $\mathscr{F}_{1}\left\lceil W_{0^{-}}\right.$ burden wv with $v \in W_{0}$.

Proof. Let $\rho=\rho_{0} \rho_{1} \rho_{2} \cdots=w^{-1} \operatorname{Play}\left(w v, \sigma^{*}, \tau\right)$. Note that $\rho_{0}=v$, which is the first vertex where the players get to choose a successor. Assume $\rho$ enters some $A_{n} \backslash U_{n-1}$. Then, it will afterwards enter $U_{n-1}$ while seeing every vertex in $A_{n} \backslash U_{n-1}$ at most once, as $A_{n}$ is an attractor and $\sigma^{*}$ behaves like an attractor strategy on $A_{n} \backslash U_{n-1}$. Now assume $\rho$ enters some $W_{0}^{n}$. Then, it will stay in $W_{0}^{n}$ until Player 1 decides to leave. However, his only choices are vertices in $A_{n-1}$, as $W_{0}^{n}$ is a trap for him in $V \backslash A_{n-1}$. Hence, once a set $A_{n} \backslash U_{n-1}$ or $W_{0}^{n}$ is left, it will never be entered again.

As $w \rho_{0}$ is an $\mathscr{F}_{1}\left\lceil W_{1}\right.$-burden, it suffices to show $\operatorname{Sc}_{F}\left(w \rho_{0} \cdots \rho_{n}\right) \leq 2$ for every $n>0$ and every $F \in \mathscr{F}_{1} \backslash W_{1}$. We will consider several cases for $F$ : remember that either $\operatorname{Sc}_{F}\left(w \rho_{0}\right)=0$ or $\operatorname{Sc}_{F}\left(w \rho_{0}\right)=1$ and $\operatorname{Acc}_{F}\left(w \rho_{0}\right)=\emptyset$.

- $F \cap\left(\bigcup_{n \geq 1}\left(A_{n} \backslash U_{n-1}\right)\right) \neq \emptyset$ : Every vertex in $\bigcup_{n \geq 1}\left(A_{n} \backslash U_{n-1}\right)$ occurs at most once in $\rho$. Hence, $\operatorname{Sc}_{F}\left(w \rho_{0} \cdots \rho_{n}\right) \leq 2$ for every $n>0$ by Lemma191.

- $F \subseteq \bigcup_{n \geq 1} W_{0}^{n}$ with $F \cap W_{0}^{i} \neq \emptyset$ and $F \cap W_{0}^{j} \neq \emptyset$ for $i<j$ : $\rho$ cannot visit $W_{j}$ after it has visited $W_{i}$. Thus, $\operatorname{Sc}_{F}\left(w \rho_{0} \cdots \rho_{n}\right) \leq 2$ for every $n>0$ by Lemma 192

- $F \subseteq W_{0}^{j}$ for some $j$ : If $\rho$ never visits $W_{0}^{j}$, then $\operatorname{Sc}_{F}\left(w \rho_{0} \cdots \rho_{n}\right)=0$ for every $n>0$. So, assume $\rho$ enters $W_{0}^{j}$ at position $\rho_{m}$ for some $m \geq 0$.

Suppose $m=0: w \rho_{0}$ is also an $\mathscr{F}_{1} \uparrow W_{0}^{j}$-burden and $w \rho_{0} \rho_{1} \rho_{2} \cdots$ is played according to $\sigma_{j}^{\mathrm{R}}$ until Player 1 decides to leave $W_{0}^{j}$ at some position $p>m$. Applying the inductive hypothesis yields that $\sigma_{j}^{\mathrm{R}}$ guarantees $\operatorname{Sc}_{F}\left(w \rho_{0} \cdots \rho_{n}\right) \leq 2$ for every $n$ in the range $m \leq n \leq p$. Should the play leave $W_{0}^{j}$, then $\mathrm{Sc}_{F}$ is reset to 0 and stays 0 , as $W_{0}^{j}$ cannot be visited again. If Player 1 never leaves $W_{0}^{j}$, then the scores are bounded by 2 throughout the whole play.

If $m>0$, then $\operatorname{Sc}_{F}\left(w \rho_{0} \cdots \rho_{n}\right)=0$ for every $n<m$. Also, the play $\rho_{m} \rho_{m+1} \rho_{m+2} \cdots$ in $W_{0}^{j}$ starts with the $\mathscr{F}_{1} \uparrow W_{0}^{j}$-burden $w \rho_{0} \cdots \rho_{m}$, (as $\rho_{m-1} \notin W_{0}^{j}$ ) and the inductive hypothesis on $\sigma_{j}^{\mathrm{R}}$ guarantees $\operatorname{Sc}_{F}\left(w \rho_{0} \cdots \rho_{n}\right) \leq 2$ until $W_{0}^{j}$ is left, from which point onwards $\operatorname{Sc}_{F}$ is always 0 .

We now turn our attention to the strategy for Player 1. For the rest of this section $n$ will be the index at which Zielonka's algorithm terminated, and $k=\operatorname{BrnchFctr}\left(\mathscr{Z}_{\mathscr{F}_{0}, \mathscr{F}_{1}}\right)$. The situation for Player 1 consists of $k$ overlapping instances, one for each child, of the situation depicted in Figure 5

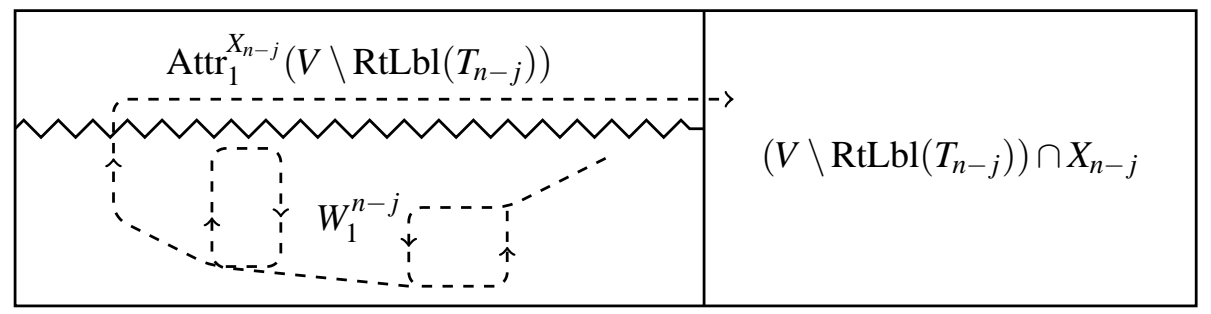

Figure 5: The structure of $W_{1}$ with respect to $T_{n-j}$. The dashed line indicates a part of a play between two change points. 
For the sake of convenience we define $Z_{j}=X_{j} \backslash \operatorname{Rt} \operatorname{Lbl}\left(T_{j}\right)$ for every $j$ in the range $n-k<j \leq n$. For every $j$ in the range $n-k<j \leq n$, we have an attractor strategy for Player 1 on $\operatorname{Attr}_{1}^{X_{j}}\left(Z_{j}\right) \backslash Z_{j}$ which we call $\tau_{j}^{\mathrm{A}}$, and we have a recursively computed winning strategy $\tau_{j}^{\mathrm{R}}$ for Player 1 on $G\left[W_{1}^{j}\right]$. Once again, we can assume the inductive hypothesis holds for the strategy $\tau_{j}^{\mathrm{R}}$, which means that $\operatorname{MaxSc}_{\mathscr{F}_{0} \mid W_{1}^{j}}\left(\operatorname{Play}\left(w v, \sigma, \tau_{j}^{\mathrm{R}}\right) \leq\right.$ 2 for every strategy $\sigma$ of Player 0 in $G\left[W_{1}^{j}\right]$ and every $\mathscr{F}_{0} \uparrow W_{1}^{j}$-burden $w v$ with $v \in W_{1}^{j}$.

Our strategy improves the strategy given by Zielonka in the sense that it uses a different method for choosing a new child of the root. Zielonka's strategy works through the children in a cyclic order, which means that when the play enters the set $V \backslash \operatorname{RtLbl}\left(T_{j}\right)$ the strategy will then move on to the child $T_{j+1}$, and begin playing either $\tau_{j+1}^{\mathrm{A}}$ or $\tau_{j+1}^{\mathrm{R}}$. By contrast, we will use a more careful method for picking the next child of the root that will be considered.

Our method for picking the next child will make its decision based on which sets of the opponent have either non-zero score or a non-empty accumulator. For this purpose, we define the indicator function of a play Ind: $V^{+} \rightarrow 2^{V}$ as

$$
\operatorname{Ind}(w)=\bigcup_{\substack{F \in \mathscr{F}_{0}: \\ \operatorname{Sc}_{F}(w)>0}} F \cup \bigcup_{\substack{F \in \mathscr{F}_{0}: \\ \operatorname{Acc}_{F}(w) \neq \emptyset}} \operatorname{Acc}_{F}(w) .
$$

Recall that Lemma 7 implies that the sets we are considering form a chain in the subset relation. This implies that the indicator function always gives some subset of a set that belongs to the opponent. Therefore, we can argue that there must always exist a child of the root whose label contains the indicator set.

Lemma 21. For every $w$, there is some $j$ in the range $n-k<j \leq n$ such that $\operatorname{Ind}(w) \subseteq \operatorname{RtLbl}\left(T_{j}\right)$.

Proof. Lemma7implies that there is a maximal set $G$ such that $\operatorname{Ind}(w)=G$, with either $\operatorname{Sc}_{G}(w)>0$ or $\operatorname{Acc}_{F}(w)=G$ for some $F \in \mathscr{F}_{0}$ with $G \subseteq F$. Hence, $\operatorname{Ind}(w) \subseteq F$ for some $F \in \mathscr{F}_{0}$, and, by definition of $\mathscr{Z}_{\mathscr{F}_{0}, \mathscr{F}_{1}}$, there is some child of the root labeled by $\operatorname{RtLbl}\left(T_{j}\right)$ such that $F \subseteq \operatorname{RtLbl}\left(T_{j}\right)$.

When a new child must be chosen, our strategy will choose some child whose label contains the value of the indicator function for the play up to that point. It is also critically important that this condition is used when picking the child in the first step. This is the part of the strategy where the finite initial path can have an effect on the decisions that the strategy makes.

We can now formally define this strategy. We begin by defining an auxiliary function that specifies which child the strategy is currently considering. We define $c: W_{1}^{*} \rightarrow\{n-k+1, \ldots, n, \perp\}$ as $c(\varepsilon)=\perp$ and

$$
c(w v)=\left\{\begin{array}{ll}
c(w) & \text { if } v \in \operatorname{RtLbl}\left(T_{c(w)}\right) \\
j & \text { if } v \notin \operatorname{RtLbl}\left(T_{c(w)}\right), \operatorname{Ind}(w v) \neq \emptyset \text { and } j \text { minimal with } \operatorname{Ind}(w v) \subseteq \operatorname{RtLbl}\left(T_{j}\right) \\
j & \text { if } v \notin \operatorname{RtLbl}\left(T_{c(w)}\right), \operatorname{Ind}(w v)=\emptyset \text { and } j \text { minimal with } v \in \operatorname{RtLbl}\left(T_{j}\right) \\
\perp & \text { if } v \notin \bigcup_{n-k<j \leq n} \operatorname{RtLbl}\left(T_{j}\right)
\end{array} .\right.
$$

Now we can define $\tau^{*}$ for $W_{1}$ as

$$
\tau(w v)^{*}=\left\{\begin{array}{ll}
\tau_{j}^{\mathrm{R}}(w v) & \text { if } c(w v)=j, v \in W_{1}^{j} \text { and } w^{\prime} \text { is the longest suffix of } w \text { with } \operatorname{Occ}\left(w^{\prime}\right) \subseteq W_{1}^{j} \\
\tau_{j}^{\mathrm{A}}(v) & \text { if } c(w v)=j, v \in \operatorname{RtLbl}\left(T_{j}\right) \backslash W_{1}^{j} \\
x & \text { if } c(w v)=\perp \text { where } x \in W_{1} \text { with }(v, x) \in E
\end{array} .\right.
$$


We will now prove that this strategy has the required properties. Our proof will use the concept of a change point, which is a position in a play where the $c$ function changes. More formally, suppose that $\rho=\rho_{0} \rho_{1} \rho_{2} \cdots=w^{-1}$ Play $\left(w v, \sigma, \tau^{*}\right)$ for some $\mathscr{F}_{0}$-burden $w v$ with $v \in W_{1}$ and $\sigma \in \Pi_{0}$. Note that $\rho_{0}=v$, which is the first vertex at which the players get to choose the successor. We say that a position $r$ of $\rho$ is a change point, if $r=0$ or if $c\left(w \rho_{0} \cdots \rho_{r-1}\right) \neq c\left(w \rho_{0} \cdots \rho_{r}\right)$.

Let $x$ be a finite prefix of an infinite play that is consistent with $\tau$ such that the last position in $x$ is a change point. Moreover, assume that $x$ satisfies the burden property. Our strategy will pick some index $j$ such that $\operatorname{Ind}(x) \subseteq \operatorname{RtLbl}\left(T_{j}\right)$. The play will then remain in the set $W_{1}^{j}$ until Player 0 chooses to leave the set $W_{1}^{j}$, at which point the strategy attracts to the set $V \backslash \operatorname{RtLbl}\left(T_{j}\right)$. Once such a vertex is reached, the scores for all sets $F \in \mathscr{F}_{0}$ with $\operatorname{Sc}_{F}(x)>0$ are reset to 0 and the accumulator for $F$ is empty for every $F \in \mathscr{F}_{0}$ with $\operatorname{Acc}_{F}(x) \neq \emptyset$. While attracting the play to $V \backslash \operatorname{RtLbl}\left(T_{j}\right)$ the scores for other sets $F \in \mathscr{F}_{0}$ might rise and the accumulators fill up. However, as every vertex in the attractor is seen at most one, we are able to show the following: if the play up to a change point is a $\mathscr{F}_{0}$-burden, then the play up to the next change point is also a burden. As a $\mathscr{F}_{0}$-burden bounds the scores of Player 0 be 2 , this suffices to prove that $\tau^{*}$ bounds Player 0's scores by 2 .

Lemma 22. Let $\rho$ be as above and let $r<s$ be two change-points such that there exists no change point $t$ with $r<t<s$. If $w \rho_{0} \cdots \rho_{r}$ is an $\mathscr{F}_{0}\left\lceil W_{1}\right.$-burden, then so is $w \rho_{0} \cdots \rho_{s}$.

Proof. From the definition of a change point we get $c\left(\rho_{0} \cdots \rho_{t}\right)=c\left(\rho_{0} \cdots \rho_{r}\right)$ for every $t$ in the range $r<t<s$.

If $c\left(w \rho_{0} \cdots \rho_{r}\right)=\perp$, then $\rho_{t} \notin \bigcup_{n-k<j \leq n} \operatorname{RtLbl}\left(T_{j}\right)$, which implies $\rho_{t} \notin F$ for every $F \in \mathscr{F}_{0}$. Hence, we have $\operatorname{Sc}_{F}\left(w \rho_{0} \cdots \rho_{t}\right)=0$ for every $r \leq t<s$ and every $F \in \mathscr{F}_{0}$. Furthermore, we have either $\operatorname{Sc}_{F}\left(w \rho_{0} \cdots \rho_{s}\right)=0$, if $F \neq\left\{\rho_{s}\right\}$ and $\operatorname{Sc}_{F}\left(w \rho_{0} \cdots \rho_{s}\right)=1$ and $\operatorname{Acc}_{F}\left(w \rho_{0} \cdots \rho_{s}\right)=\emptyset$ otherwise.

Now, assume $c\left(w \rho_{0} \cdots \rho_{r}\right)=j$ for some $j$ in the range $n-k<j \leq n$. Then, there exists an $u$ in the range $r \leq u \leq s$ such that $\rho_{r} \cdots \rho_{u-1}$ is in $W_{1}^{j}, \rho_{u} \cdots \rho_{s-1}$ is in $\operatorname{Attr}_{1}^{X_{j}}\left(Z_{j}\right) \backslash Z_{j}$, and we have $\rho_{s} \notin \operatorname{RtLbl}\left(T_{j}\right)$. Note that both parts could be empty. The situation is depicted in Figure 6 (cf. also Figure 5).

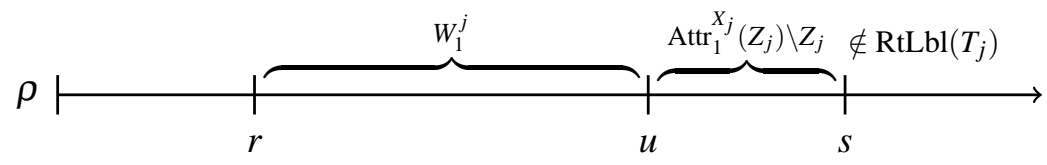

Figure 6: A part of a play between two change points.

Furthermore, at positions $i$ in the range $r \leq i \leq u-1$, Player 1 plays according to $\tau_{j}^{\mathrm{R}}$ and positions $i$ in the range $u \leq i \leq s-1$, he plays according to $\tau_{j}^{\mathrm{A}}$. This implies that every vertex in $\operatorname{Attr}_{1}^{X_{j}}\left(Z_{j}\right) \backslash Z_{j}$ is seen at most once in between $\rho_{r}$ and $\rho_{s}$, i.e., in the infix $\rho_{u} \cdots \rho_{s-1}$.

Finally, let $\operatorname{Ind}\left(w \rho_{0} \cdots \rho_{r}\right)=G$. If $G \neq \emptyset$, then $G \subseteq \operatorname{RtLbl}\left(T_{j}\right)$; otherwise, $\rho_{r} \in \operatorname{RtLbl}\left(T_{j}\right)$, both by definition of $c$.

It suffices to show for every $F \in \mathscr{F}_{0}\left\lceil W_{1}\right.$ :

1. If $\operatorname{Sc}_{F}\left(w \rho_{0} \cdots \rho_{r}\right)=1$ and $\operatorname{Acc}_{F}\left(w \rho_{0} \cdots \rho_{r}\right)=\emptyset$, then $\operatorname{Sc}_{F}\left(w \rho_{0} \cdots \rho_{t}\right) \leq 2$ for all $r<t<s$ and $\mathrm{Sc}_{F}\left(w \rho_{0} \cdots \rho_{s}\right)=0$.

2. If $\operatorname{Sc}_{F}\left(w \rho_{0} \cdots \rho_{r}\right)=0$, then $\operatorname{Sc}_{F}\left(w \rho_{0} \cdots \rho_{t}\right) \leq 2$ for all $r<t<s$ and either $\operatorname{Sc}_{F}\left(w \rho_{0} \cdots \rho_{s}\right)=1$ and $\operatorname{Acc}_{F}\left(w \rho_{0} \cdots \rho_{s}\right)=\emptyset$ or $\operatorname{Sc}_{F}\left(w \rho_{0} \cdots \rho_{s}\right)=0$.

1. As $\emptyset \neq F \subseteq \operatorname{Ind}\left(w \rho_{0} \cdots \rho_{r}\right)$, we have $F \subseteq \operatorname{RtLbl}\left(T_{j}\right)$ and hence $\operatorname{Sc}_{F}\left(w \rho_{0} \cdots \rho_{s}\right)=0$, as $\rho_{s} \in Z_{j}=$ $X_{j} \backslash \operatorname{RtLbl}\left(T_{j}\right)$. It remains to show $\operatorname{Sc}_{F}\left(w \rho_{0} \cdots \rho_{t}\right) \leq 2$ for all $r<t<s$. We consider several cases for $F$ : 
- $F \cap Z_{j} \neq \emptyset$ : as the vertices in $Z_{j}$ are not visited by $\rho_{r} \cdots \rho_{s-1}$, the score of $F$ cannot increase in this interval.

- $F \cap \operatorname{Attr}_{1}^{X_{j}}\left(Z_{j}\right) \backslash Z_{j} \neq \emptyset$ : every vertex in the attractor is seen at most once. Hence, we obtain $\mathrm{Sc}_{F}\left(w \rho_{0} \cdots \rho_{t}\right) \leq 2$ for all $r<t<s$ by Lemma191.

- $F \subseteq W_{1}^{j}$ : If $\rho_{r} \in \operatorname{Attr}_{1}^{X_{j}}\left(Z_{j}\right) \backslash Z_{j}$, then $\operatorname{Sc}_{F}$ is reset to 0 at $\rho_{r}$ and stays 0 until $\rho_{s}$, hence, we have $\operatorname{Sc}_{F}\left(w \rho_{0} \cdots \rho_{t}\right) \leq 2$ for all $r<t<s$.

So, suppose $\rho_{r} \in W_{1}^{j}$. As $w \rho_{0} \cdots \rho_{r}$ is also an $\mathscr{F}_{0} \uparrow W_{1}^{j}$ - burden and as $F \in \mathscr{F}_{0} \uparrow W_{1}^{j}$, the inductive hypothesis on $\tau_{1}^{j}$ guarantees $\operatorname{Sc}_{F}\left(w \rho_{0} \cdots \rho_{t}\right) \leq 2$ for every $r<t<u$. As $\rho_{t} \notin W_{1}^{j}$ for every $t$ in the range $u \leq t<s$, we also have $\operatorname{Sc}_{F}\left(w \rho_{0} \cdots \rho_{t}\right)=0$ for these positions.

2. Let $G=\operatorname{Acc}_{F}\left(w \rho_{0} \cdots \rho_{r}\right) \subseteq \operatorname{Ind}\left(w \rho_{0} \cdots \rho_{r}\right)$. Note that $G \subseteq \operatorname{RtLbl}\left(T_{j}\right)$, but it could be the case that $F \nsubseteq \operatorname{RtLbl}\left(T_{j}\right)$. Again, we consider several cases for $F$ :

- If $\rho_{s} \in F$, then $\rho_{s} \notin \operatorname{Acc}_{F}\left(w \rho_{0} \cdots \rho_{r}\right)$, as $\operatorname{Acc}_{F}\left(w \rho_{0} \cdots \rho_{r}\right) \subseteq \operatorname{RtLbl}\left(T_{j}\right)$ and $\rho_{s} \notin \operatorname{RtLbl}\left(T_{j}\right)$. Hence, $\mathrm{Sc}_{F}$ stays 0 at every position between $r$ and (excluding) $s$, as the vertex $\rho_{s}$ is never visited. If $\operatorname{Sc}_{F}\left(w \rho_{0} \cdots \rho_{s}\right)=1$, then $\operatorname{Acc}_{F}\left(w \rho_{0} \cdots \rho_{s}\right)=\emptyset$; otherwise $\operatorname{Sc}_{F}$ is 0 at position $s$, too.

- If $\rho_{s} \notin F$, then $\operatorname{Sc}_{F}\left(w \rho_{0} \cdots \rho_{s}\right)=0$. To bound the score between the positions $r$ and $s$ by 2, we have to consider three subcases: either $F \cap Z_{j} \neq \emptyset, F \cap \operatorname{Attr}_{1}^{X_{j}}\left(Z_{j}\right) \backslash Z_{j} \neq \emptyset$ or $F \subseteq W_{1}^{j}$. All cases can be solved by analogous reasoning to these cases in 1

Now, to prove the inductive step for $W_{1}$, we simply need to observe that the finite path ending at the first change point is a burden by assumption.

Lemma 23. We have $\operatorname{MaxSc}_{\mathscr{F}_{0}\left\lceil W_{1}\right.}\left(\operatorname{Play}\left(w v, \sigma, \tau^{*}\right)\right) \leq 2$ for every strategy $\sigma \in \Pi_{0}$ and every $\mathscr{F}_{0}\left\lceil W_{1^{-}}\right.$ burden wv with $v \in W_{1}$.

Proof. Let $\rho=w^{-1} \operatorname{Play}\left(w v, \sigma, \tau^{*}\right)$. If $\rho$ contains infinitely many change points, then Lemma22 implies $\operatorname{MaxSc}_{\mathscr{F}_{0}}\left(\operatorname{Play}\left(w v, \sigma, \tau^{*}\right)\right) \leq 2$ as the play starts with a burden, i.e., there scores are bounded by 2 in $w v$, and in between any two change points, the scores are bounded by 2 as well. If $\rho$ contains only finitely many change points, then Lemma 22 implies that the scores of Player 0 up to the last change point are bounded by 2 . From that point onwards, Play $\left(w v, \sigma, \tau^{*}\right)$ is consistent with some $\tau_{1}^{j}$, and the play up to that point is a $\mathscr{F}_{1} \uparrow W_{1}^{j}$-burden, as it is an $\mathscr{F}_{1} \uparrow W_{1}$-burden due to Lemma22 Hence, the scores for every set $F \in \mathscr{F}_{1} \backslash W_{1}^{j}$ are bounded by 2 from that point onwards, by the inductive hypothesis on $\tau_{1}^{j}$. The scores of every $F \in \mathscr{F}_{1} \backslash W_{1}$ with $F \nsubseteq W_{1}^{j}$ are bounded by 1 , as vertices not in $W_{1}^{j}$ are no longer visited.

Finally, we can prove Lemma[15, which also completes the proof of Theorem 14

Proof. Theorem 12 yields that algorithm 11 is correct, i.e. the sets $W_{i}$ returned are indeed the winning regions of the players. We prove the following stronger statement by induction over the height of $\mathscr{Z}_{\mathscr{F}_{0}, \mathscr{F}_{1}}$ : let $V$ be the vertex set of $G$. Player $i$ has a winning strategy $\sigma$ for her winning region $W_{i}$ in $G$ such that $\operatorname{MaxSc}_{\mathscr{F}_{1-i} \backslash V}(w v, \sigma, \tau) \leq 2$ for every strategy $\tau \in \Pi_{1-i}$ and every $\mathscr{F}_{1-i}\lceil V$-burden $w v$ in $G$. This implies Lemma 15 as the finite play $v$ for every $v \in W_{i}$ is an $\mathscr{F}_{1-i} \uparrow V$-burden.

For the induction start, apply Lemma 18, In the induction step, use the strategies obtained from the induction hypothesis to define $\sigma^{*}$ and $\tau^{*}$ as above and apply Lemma 20 respectively Lemma 23. Both strategies are winning, as they bound the scores of the opponent by 2 . 


\section{Conclusion}

We have presented a criterion to stop plays in a Muller game after a finite amount of time that preserves winning regions. Our bound $3^{|G|}$ on the length of a play improves the bound $|G| !+1$ obtained by a reduction to parity games. Furthermore, our techniques show that the winning player can bound the scores of the opponent by 2 and that this bound 2 is tight.

However, it remains open whether a play can also be stopped after a score of 2 is reached. As the winning player cannot always avoid a score of 2 for the opponent, one has to show that the winning player always reaches a score of 2 for one of her sets before the opponent reaches score 2 for one of his sets. Our approach does not seem to be suitable for this, as the notion of a burden is not sufficient for this goal. Furthermore, it is unclear how to strengthen the definition while still retaining Lemmata corresponding to Lemma 20 and Lemma 23

A finite-time Muller game with threshold $k$ is a reachability game (in the unraveling of the original arena up to depth at most $k^{|G|}$ ), which can be solved with simple algorithms. Another interesting direction for research is to find a construction which turns a winning strategy for a finite-time Muller game with threshold 3 (or 2, if it is equivalent) into a finite-state strategy for the original Muller game. It is conceivable that such a construction would yield memory structures that are optimized for a given arena, something which does not hold for the LAR respectively Zielonka tree structures.

Acknowledgements. The authors want to thank Wolfgang Thomas for bringing McNaughton's work to their attention and Marcus Gelderie, Michael Holtmann, Marcin Jurdziński, and Jörg Olschewski for fruitful discussions on the topic. Also, they want to thank the referees for their helpful comments.

\section{References}

[1] Julius R. Büchi \& Lawrence H. Landweber (1969): Solving Sequential Conditions by Finite-state Strategies. Trans. Amer. Math. Soc. 138, pp. 295-311.

[2] Krishnendu Chatterjee, Thomas A. Henzinger \& Florian Horn (2009): Finitary winning in omega-regular games. ACM Trans. Comput. Log. 11(1). Available at http://doi.acm.org/10.1145/1614431. 1614432

[3] Stefan Dziembowski, Marcin Jurdziński \& Igor Walukiewicz (1997): How Much Memory is Needed to Win Infinite Games? In: LICS, pp. 99-110. Available at http://www.computer.org/proceedings/lics/ 7925/79250099abs.htm

[4] Stefan Dziembowski, Marcin Jurdziński \& Igor Walukiewicz (1998): How Much Memory is Needed to Win Infinite Games? Available at http://www.dcs.warwick.ac.uk/ mju/Papers/DJW98-memory.ps Unfinished draft of [3].

[5] Yuri Gurevich \& Leo Harrington (1982): Trees, Automata, and Games. In: STOC, ACM, pp. 60-65.

[6] Florian Horn, Wolfgang Thomas \& Nico Wallmeier (2008): Optimal Strategy Synthesis in Request-Response Games. In: Sung Deok Cha, Jin-Young Choi, Moonzoo Kim, Insup Lee \& Mahesh Viswanathan, editors: ATVA, Lecture Notes in Computer Science 5311, Springer, pp. 361-373. Available at http://dx.doi. org/10.1007/978-3-540-88387-6_31

[7] Robert McNaughton (1993): Infinite Games Played on Finite Graphs. Ann. Pure Appl. Logic 65(2), pp. 149-184.

[8] Robert McNaughton (2000): Playing Infinite Games in Finite Time. In: Arto Salomaa, Derick Wood \& Sheng Yu, editors: A Half-Century of Automata Theory, World Scientific, pp. 73-91. 
[9] Ernst Zermelo (1913): Über eine Anwendung der Mengenlehre auf die Theorie des Schachspiels. In: Proc. of the Fifth Congress of Mathematicians, Vol. 2, Cambridge Press, pp. 501-504.

[10] Wieslaw Zielonka (1998): Infinite Games on Finitely Coloured Graphs with Applications to Automata on Infinite Trees. Theor. Comput. Sci. 200(1-2), pp. 135-183. Available at http://dx.doi.org/10.1016/ S0304-3975 (98) 00009-7

[11] Martin Zimmermann (2009): Time-Optimal Winning Strategies for Poset Games. In: Sebastian Maneth, editor: CIAA, Lecture Notes in Computer Science 5642, Springer, pp. 217-226. Available at http://dx. doi.org/10.1007/978-3-642-02979-0_25 